\title{
Factors affecting adherence to asthma treatment: patient and physician perspectives
}

\author{
Eric Van Ganse, Ann-Christin Mörk, Liesl M Osman, Paul Vermeire, Laurent Laforest, Alexia Marrel, \\ Elisabeth Ståhl
}

\begin{abstract}
Aims: To identify important factors affecting treatment adherence of patients with asthma and to summarise this information as a guide for physicians.
\end{abstract}

Methods: Information from literature and interviews with 12 respiratory physicians (four each from France, Spain and UK) and 46 asthma patients was obtained. Factors affecting adherence to asthma treatment were identified, reviewed and a flow chart developed to indicate the relationship between key factors.
Results: Major factors influencing adherence included: the patientphysician relationship; the patient's understanding of the disease and its treatment; the patient's beliefs and perception of the disease and its treatment, and, importantly, the patient's willingness to take an active part in his/her asthma management.

Conclusion: Patient adherence to asthma can be improved, and the likelihood of treatment success increased, by paying attention to the factors that influence patients' willingness to participate in their treatment.

Key words: Asthma, adherence, patient beliefs, medication

\section{Eric van Ganse}

CHU-Lyon

\section{Ann-Christin Mörk}

AstraZeneca, Sweden

\section{Liesl M Osman}

University of Aberdeen

\section{Paul Vermeire}

University of Antwerp

\section{Laurent Laforest}

CHLU-Lyon

\section{Alexia Marrel}

Mapi Values, Lyon

\section{Elisabeth Ståh}

AstraZeneca, Sweden

Correspondence to:

Dr Eric van Ganse

Centre Hospitalier Lyon Sud

(CHLS)

Unité de

Pharmacoépidemiologie

Sainte Eugénie (bâtiment 5F)

69495 Pierre-Bénite

Cedex France

Tel +3347266 6438

Fax +33472666444

Email:

eric.vanganse@chulyon.fr

Date submitted: $26 / 01 / 03$ Date Accepted: 27/05/03

Prim Care Resp J 2003; 12(2):46-51

\section{Introduction}

Adherence $\uparrow$ to treatment in asthma is mostly poor and may be a significant factor contributing to adverse outcomes, reduced health-related quality of life (HRQL) and high costs of the disease. ${ }^{1}$ There are many reasons for poor adherence, including beliefs about medications, which may often be at variance with current scientific evidence. ${ }^{2}$ It is important to understand the ways in which patients make their decisions to adhere to treatment, as this may be directly related to the benefits they obtain from treatment. ${ }^{3}$

In this article, information from a literature review and interviews from a small group of European respiratory physicians and asthma patients are used to identify patient and physician views on the important factors involved in treatment adherence. The aim was to process this information as a guide for physicians to assist them in improving the management of patients, more particularly by improving adherence.

\section{Methods}

Articles published from 1990 onwards with abstracts in French or English were identified from Medline or Excerpta Medica databases (keywords were asthma and patient compliance/adherence). These articles were screened for relevance based on the content of their abstracts. Individual factors affecting adherence were identified from individual studies measuring adherence and from previous review articles. An expert group, consisting of an epidemiologist and two respiratory physicians, reviewed and categorised the factors identified as influencing adherence and interrelated these in a flow chart.

Twelve respiratory physicians (four each from France, Spain and UK) with experience of treating asthma patients were selected from a database of physicians maintained by Mapi Values and interviewed by a
Clinical Research Associate (France, Spain) or a physician (UK). Interviews were carried out by phone in France and the UK and face-to-face in Spain. Physicians' views were obtained using a semistructured interview guide; this was structured, to obtain information on: asthma, diagnosis, cause, treatment (medications and otherwise); patientmanagement practices; the impact of asthma on patients' lives; patient understanding of asthma and adherence; overall perspective. The physicians provided opinion on the preliminary flow chart and suggested changes either verbally or by annotation of the chart. The chart was faxed to physicians who were interviewed by phone, and annotated charts returned similarly.

Face-to-face interviews were conducted with asthma patients referred by respiratory physicians (these included those interviewed) and general practitioners, who supplied details of their patient's treatments and disease status according to Global Initiative for Asthma (GINA) guidelines. ${ }^{4}$ Inclusion criteria were predefined. Patients were required to have asthma and have been treated with inhaled corticosteroids (ICS) with or without a long-acting $\beta 2$-agonist (LABA) as controller medication ( $\geq 2$ years). It was intended that half of the patients would have had a recent asthma exacerbation (use of oral steroids within 6 months or a hospitalisation/emergency room visit within 2 years), a similar number would be male and female, and at least 18 patients (half male and female) would be aged between 20 and 35 years.

Their physician offered patients the opportunity to volunteer for the study. An explanation of the purpose of the study was given to the patients who volunteered by the interviewer, and verbal agreement was obtained before commencing the interview.

Clinical Research Associates carried out interviews with the patients using a semi-structured interview guide, structured to obtain information and views on the following: socio-demographic characteristics; 
patient knowledge of asthma; details of their current treatment; advantages and disadvantages of their treatment; purpose of treatments (e.g. differences between relievers and controllers); adherence to treatment (and reasons for non-adherence); their role in treatment; information provided on asthma and treatment; overall expectations and satisfaction with treatment and the management of their disease; information provided; impact of asthma on their lives; overall satisfaction with treatment. Patients were asked to rate the severity of their asthma as either mild, moderate, severe or very severe. The measure of agreement between patient ratings of asthma severity and the physician-assigned severity of asthma (GINA stage) was tested using a weighted kappa test.

Socio-demographic data from respiratory physicians and patients, and clinical data were analysed by descriptive statistics. Verbatim responses were tabulated from interviews and reviewed by the expert group, who subsequently revised the flow chart.

\section{Results}

\section{Literature review}

From 971 articles identified from the Medline and Excerpta Medica databases, 493 individual articles were identified with abstracts in English or French. Of these, 275 articles containing relevant information on factors affecting adherence were identified. The individual factors suggested or demonstrated to be associated with adherence in the articles reviewed, and any additions from subsequent expert review, are grouped in categories in Table 1 .

\section{Physician interviews}

Physicians (11 male, one female) were aged between 33 and 55 years (mean 42 years). Types of practice were: hospital, five; private, two; both, five. On average each physician saw 24 patients per day (range 5-60), which included six (range 2-12) asthma patients.

\section{Diagnosis and choice of treatment}

All physicians based their diagnosis on medical history, symptoms and examination. Diagnosis was usually confirmed by tests ( $\mathrm{n}=9$ ), typically lungfunction measurements, and sometimes supported with other tests, e.g. allergy skin testing $(\mathrm{n}=2)$ and blood tests $(n=2)$. Family history was the only factor commonly cited by physicians $(\mathrm{n}=8)$ as a determinant of asthma. The adverse effects of asthma on HRQL and relationship with severity of disease were generally recognised. The most frequently cited negative impacts of asthma were related to patients' work and scholastic activities $(\mathrm{n}=11)$; others included effects on sleep $(n=3)$, restrictions on exercise, social and leisure activities $(n=3)$, and partner/colleague relationships $(\mathrm{n}=1)$. As well as clinical status, the physician's choice of treatment took into account patient wishes $(\mathrm{n}=8)$, their intellectual ability $(\mathrm{n}=2)$, and ability to use an inhaler $(\mathrm{n}=6)$; the latter was considered a particular problem in the elderly $(n=5)$.

\section{Table 1. Factors that may affect patient adherence to asthma treatment.}

*Categories of factors considered of greatest importance

Patient characteristics: Age; gender; educational level; socio-economic level; ethnic origin; physical ability; comorbid diseases

Environment and support: Attitudes and involvement/support of family/friends social relationships; lifestyle

Patient knowledge and understanding of asthma:* Asthma, symptom recognition, understanding severity and variability of symptoms; how to manage symptoms; triggers; medications

Psychological state: Anxiety; depression; stress; forgetfulness

Beliefs/attitudes and perception of disease/treatment:* Factors that control health; locus of control - internal (patient controls their own destiny), self-efficacy (ability to manage the disease themselves) and external (something or someone else has control); fate (chance); helplessness - neither he nor she or the physician can do anything to help. Cultural beliefs. Perceptions of control - feeling the disease is well controlled; severity of the disease; necessity for and success/benefit of medication; perceived success of treatment; the burden of treatment (cost, complexity, social embarrassment); the medication (side-effects, dependence, fear of steroids, general beliefs)

Patient's willingness:* Desire to take active part in the management of disease; denial and rejection of diagnosis; laziness; disturbance/limitation of normal daily and social/recreational activities

Medication: Frequency of dosing; integration with other medications; simplicity of directions and use; taste; degree and nature of side effects; convenience and embarrassment in using; cost

Patient-physician relationship:* Communication; patient satisfaction with relationship with physician; confidence in physician skills; therapeutic alliance (patient takes active part in treatment decision); patient expectations; patient information (on the disease, on the treatment, on the device); continuity of care; failure to analyse medication behaviours; physician training in communication skills; inappropriate medication or dose prescribed.

Specialist-general practitioner relationship: Consistency of advice and treatment; trust and mutual respect

Access to medical care and advice: Location of amenities; financial situation (cost of medication and transport); availability of medication; availability of educational literature

Severity and duration of the disease: Ability to cope with symptoms; motivation to take medication; length of therapy; experiences of previous medications

\section{Adherence and efficacy of treatment}

Estimates of adherence to treatment of their asthma patients varied widely between physicians; ratings for the percentage of patients who were non-adherent were highest in the UK (France, 10-50\%; Spain, 1550\%; UK, 30-70\%). Physicians expressed a wide range of opinions towards adherence and efficacy of treatments, which are summarised in Table 2. All but one physician felt that the fear of side effects or addiction outweighed efficacy benefits as influencing factors for stopping treatment. All physicians considered patient beliefs and perceptions of disease were important issues in adherence.

\section{Review of the flow chart}

The physicians all agreed with the flow chart presented; however, six physicians suggested the following additional items: general practitionerspecialist relationship $(n=2)$, nurse-patient relationship; family attitude; use of explanatory leaflets; severity of asthma $(n=2)$; health system (availability, access, cost) $(n=2)$; concomitant disease; use of peak flow meters.

†Adherence
Adherence was defined as the
extent to which patient's
behaviour with respect to their
treatment for asthma coincides
with the clinical treatment
intended by the physician. The
term "adherence" is used
throughout in this article to
imply a voluntary choice of the
patient in deciding whether or
not to follow a treatment plan
offered to them by the
physician. The term
"compliance", though frequently
used interchangeably with
"adherence", is less preferred as
it implies a passive submission
to the prescriber's instruction."


For personal use only.

Not to be reproduced without the permission of the Primary Care Respiratory Journal

Table 2. Opinions of physicians regarding adherence and efficacy of treatment.

\section{Number of physicians (of 12)*}

\section{Reasons why patients stop treatment:}

side-effects

feeling better

forgetfulness

treatment inappropriate or of no benefit

general dislike of medication

fear of addiction

embarrassment

psychiatric illness

Factors motivating patients to adhere to medication:

perceived efficacy

understanding the reasons for adherence

ease of use medication

having severe asthma

low cost

adverse experience of other asthma sufferers

a good physician-patient relationship

Main reasons for inefficacy of inhaled treatment:

failure to master the use of the inhaler

3

3

2

1

1

poor adherence

inappropriate treatment for severity

ineffective medication

insufficient dose

wrong diagnosis

Methods used to improve efficacy:

change the type of device

check how the patient uses treatment and consider alternatives

check for adherence

(re-)educate the patient

increase dosage

re-evaluate the patient

Actions taken if non-adherence is suspected:

provide information, advice and stress importance of adherence 6 with maintenance medication

prescribe combination inhalers

encourage the patient

make sure the patients know how to take their medication

provide written advice

suggest that, if the patient was not happy with his/her

treatment, he/she should go and see another doctor

*number of physicians who expressed the opinion without prompting

\section{Table 3. Patient characteristics}

\section{Socio-demographic characteristics}

Nationality Number (French/Spanish/British)

Age (y)

Mean (range)

$34(18-53)$

Gender (male/female) Total

$20 / 26$

Between ages 20 and $35 \quad 10 / 15$

With recent asthma exacerbation $^{\mathrm{a}} \quad 10 / 17$

Occupational status Working

Student

Retired due to asthma

Other or missing data

Educational level Elementary

Secondary

College

Postgraduate or other

Family status

Living with partner/family

Living alone

Present/past/never smoked

Smokers

Yes/No

Living with

\section{Clinical characteristics (according to physicians)}

Duration of asthma (y); mean (range)

Duration of treatment with ICS (y); mean (range)

Use of oral steroids within last 6 months (yes/no)

Hospitalisation within last 24 months (yes/no)

Emergency department treatment within the last

Other disease (yes/no)

Current asthma controller medication:

ICS (budesonide/ beclomethasone/

fluticasone/ undefined)

ICS plus LABA (separate and combination)

Current asthma reliever medication:

Short-acting 32 -agonist (salbutamol/terbutaline)

Ipratropium/salbutamol combination

ICS = inhaled corticosteroid; LABA = long-acting $\beta 2$-agonist

a. Use of oral steroids within 6 months or a hospitalisation/emergency room visit within 2 years

b. One patient was included with a duration of asthma of 1 year, because he/she had experienced emergency room treatment

c. Duration of ICS treatment was unknown in several Spanish patients, hence values of 0 were assigned

d. Data not provided for all patients

\section{Patient interviews}

Information on socio-demographics and clinical characteristics of patients (supplied by physicians) are summarised in Table 3. Although there was a statistically significant agreement between patient perceptions of their asthma severity (mild-very severe) and severity of asthma (GINA stages) assigned by the physician (weighted kappa $0.53, \mathrm{p}<0.001$ ), there were several instances of disagreement for individual patients (Table 4). Most patients defined asthma in terms of the symptoms and how they felt, although 14 patients attempted to explain their asthma in terms of lung function, as explained by their physicians. Only four patients mentioned inflammation. Most of them appreciated the widespread occurrence and increasing prevalence of asthma. Allergy $(n=20)$ and heredity $(\mathrm{n}=17)$ were the two most frequently mentioned causes of asthma. Most frequent triggers stated (often multiple) included dust and feathers $(\mathrm{n}=24)$; smoking $(\mathrm{n}=17)$; stress $(\mathrm{n}=17)$; air quality $(\mathrm{n}=16)$, weather $(\mathrm{n}=16)$; animal fur $(\mathrm{n}=16)$; pollen $(\mathrm{n}=15)$; and a respiratory infection (cold/bronchitis) $(\mathrm{n}=7)$.

Most patients recognised the different roles and benefits of the reliever and controller in treatment. Of 44 respondents, given a choice of medication, 32 chose the reliever and 10 the controller; and 2 insisted that both were needed. Almost half of the patients reported at times both forgetting and stopping controller treatment for a variety of reasons (Table 5).

Most patients $(\mathrm{n}=28)$ thought their first treatment would be temporary, and of these 23 considered that 
Original research

medication would cure them. Only six patients reported that their physician kept them well informed. Most had received instruction on the use of the inhalers (by physician 26; pharmacist, 3; nurse 10; a friend, 1) and three reported not having received any instructions. An equal number of patients $(n=10)$ mentioned that they had or had not discussed pros and cons of treatment with their physician. Nevertheless, most patients expressed confidence in their physicians, and only three patients were dissatisfied with the medical help they received. Most patients $(n=36)$ considered they were capable of managing their asthma themselves. Seven patients deplored the lack of psychological support, whereas 38 patients felt that they had sufficient support from their families and friends, although four patients did not discuss their asthma with them.

\section{Derivation of the final flow chart}

Additional categories of factors (medication; specialist-general practitioner relationship; disease severity) were added to finalise the flow chart after consideration of the views of physicians and patients (Figure 1).

\section{Discussion}

Information from an extensive literature review and interviews with 12 respiratory physicians and 46 patients with asthma confirmed a high frequency of inadequate controller use and identified many individual patient- and medication-related factors that influenced adherence. The inter-relationship between potentially influencing factors is complex, and, we did not attempt to rank individual factors with regard to their relative importance in positively or negatively influencing adherence to treatment. Nevertheless, we identified four categories of factors as being of the most importance in influencing adherence to treatment: (1) the patient-physician relationship, (2) patient understanding of the disease and its treatment, (3) patient's beliefs/attitudes and perception of disease/treatment and (4) patient willingness (to take an active part in asthma management).

Overall, patients interviewed had good knowledge of their asthma, trigger factors and treatment and felt there were advantages for the use of controller medications; nevertheless, in many cases patients decided to stop treatment because of inconvenience and lack of perceived benefit. Results of this study confirm the necessity for patients to appreciate the benefits of regular controller treatment and to make the medications simple and convenient to use.

\section{Although side effects were a common cause of} concern, they were not a commonly expressed reason for stopping treatment. Interestingly, some patients cited the lack of an immediate bronchodilating effect as a reason for their perception of inefficacy of controller medication. In this respect, it is possible that a single inhaler containing a combination of a rapid- and long-acting $ß 2$-agonist and an ICS may help to encourage regular therapy. Unfortunately,
Table 4. Classification of patients according to physician assessments of the GINA stage of asthma severity ${ }^{4}$ and severity as perceived by patients ${ }^{a}$

\begin{tabular}{lccccc} 
Patients' ratingb & \multicolumn{5}{c}{ GINA stage } \\
No. of & $\mathbf{1}$ & $\mathbf{2}$ & $\mathbf{3}$ & $\mathbf{4}$ \\
Matients & 12 & 6 & 4 & 1 & 1 \\
Moderate & 16 & 1 & 12 & 2 & 1 \\
Severe & 9 & 0 & 2 & 2 & 5 \\
Very severe & 3 & 0 & 0 & 0 & 3
\end{tabular}

a. Complete information was not obtained for all patients

b. There was a significant agreement between patients' rating and GINA stage (weighted kappa $=0.53, \mathrm{p}<0.001)$

Table 5. Patient interview responses regarding their medication

\begin{tabular}{|c|c|c|c|}
\hline Reliever & n & Controller & $\mathbf{n}$ \\
\hline $\begin{array}{l}\text { Use of reliever } \\
\text { when having an attack }\end{array}$ & 29 & Use of controller & 3 \\
\hline before exercise & 5 & twice daily & 29 \\
\hline before going to bed & 5 & daily & 2 \\
\hline preventing an attack & 2 & infreqently & 1 \\
\hline
\end{tabular}

after too much exercise

after smoking a lot

Advantages/Disadvantages

Benefit outweighs disadvantages $38 \quad$ Benefit outweighs disadvantages 38

$\begin{array}{llll}\text { Benefits did not outweigh } & 2 & \text { Benefits did not outweigh } & 4\end{array}$

disadvantages

\section{Advantages}

helps breathing and prevents need 29

for emergency treatment

provides immediate benefit

user-friendly

easy to carry

opens the airways

straightforward treatment

Disadvantages

none

disadvantages

\section{Advantages}

easy to use

relieves symptoms

helps control asthma

none

side effects

dependence

difficult to use

embarrassment

inefficient

\section{Disadvantages}

side effects

no immediate benefit

contains cortisone/steroids

need to take every day

too many medications

none

embarrassment

dependence

\section{Concerns about side effects}

\section{none}

cough/dry mouth/after taste

Concerns about side effects

shaking

tachycardia

weight gain

mouth ulcers 7 mouth ulcers

can weaken bones

weight gain

shivering

itch

Reasons for non-adherence with controller treatment
Forgetting $(\mathbf{n}=\mathbf{2 5})$

work (time/stress)

when feel good or symptoms

not bad

no particular reason

weekends

away from home

see no benefit in taking it
Deciding to stop treatment $(n=19)$

7 Didn't need it/felt fine

6 fear of side effects physician request

5 too complicated

3 to see how I coped

3 too many treatments

2 asthma not serious

travelling no money
$\mathrm{n}=$ the number of patients who expressed the opinion. As not all patients provided an answer
to every question, the sum of the answers is not always 46 for each question. Furthermore,

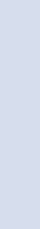

to every question, the sum of the answers is not always 46 for each question. Furthermore,

multiple answers (occasionally given) were given equal weight 
previous attempts to improve compliance by combining short-acting 32 -agonists and ICS have been unsuccessful in improving compliance. ${ }^{5,6}$ In contrast, however, improved compliance with regular ICS use has been demonstrated when a long-acting B2-agonist was added to ICS maintenance therapy.

Patients will inevitably balance potential risks against the benefits to be obtained from medications when deciding whether or not to take their medications as prescribed; non-adherence may be a logical attempt to moderate the perceived risks by taking less. ${ }^{8}$ The physician has a key role in making sure that the patient's informed choice is not made on mistaken beliefs, either about the disease or the medication. Patients may have very different viewpoints on what the objective of asthma therapy is and their level of asthma control; this is illustrated by the discrepancy between patient perceptions of severity and physicianassessed severity reported in a large European survey. ${ }^{9}$

There were also several instances in the present study of differences between patients' assessment of their own asthma severity (mild-very severe) and physicians' assessment of asthma severity (GINA stage). If patients underestimate their disease severity, it is likely that they will also underestimate the need for treatment. Educating patients about their disease and role of medication is important for improving adherence; however, good knowledge alone is no guarantee of good adherence. ${ }^{10}$ Communication skills of the physician are clearly important, and there is evidence that training physicians can improve patient self-management. ${ }^{11}$ To achieve patient willingness to actively participate in the effective treatment of the disease the physicians' approach should be adapted depending on the individual circumstances, beliefs and attitudes of the patient. Furthermore, in aiming to achieve willingness, the physician should not lose sight of clinical outcome. As Chapman et al (2000) ${ }^{12}$ state "it is better to achieve complete asthma control with an effective medication taken less than that prescribed than it is to achieve perfect compliance

\section{Figure 1. Inter-relationship of factors influencing adherence to treatment}

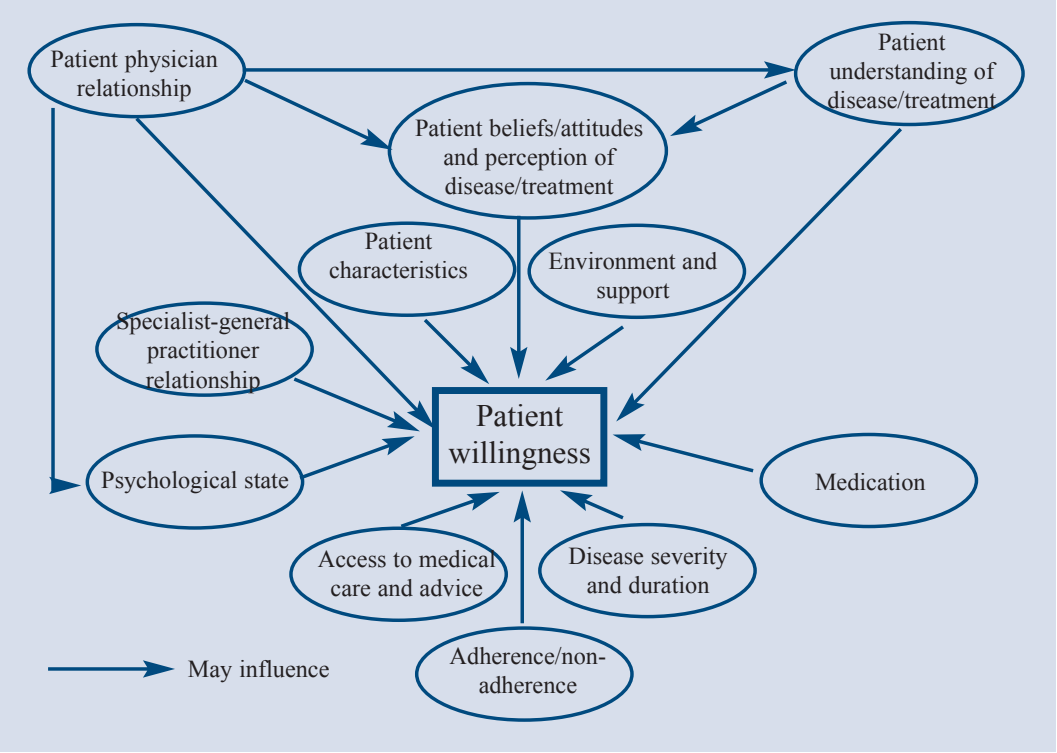

with an ineffective medication that fails to control the disease". Thus adherence and effectiveness of medications are both important.

The patients interviewed in the study were not selected at random, and it is possible that physicians chose patients with whom they were most familiar and had a good patient-physician relationship; a factor that would be expected to improve adherence. It is possible, therefore, that the population sampled may not have been fully representative of the general asthma patient population, i.e. more adherent. However, as many patients interviewed reported poor adherence, the numbers were considered sufficient to provide a general overview on factors affecting adherence from the patient perspective, and this was not considered a major limitation of the study.

The small numbers of patients and physicians interviewed precluded quantitative ratings of the importance of individual factors to be assigned and did not allow inter-country differences in practices and opinions to be evaluated; more controlled studies involving larger numbers are needed for this.

Although the respiratory specialists provided their opinions from a broad perspective, the inclusion of general practitioners and respiratory nurses in future surveys would increase the credibility of these findings for primary care. Furthermore, as we only interviewed adults with asthma, specific factors influencing adherence in childhood asthma may not have been identified. For younger children the parents have a key-influencing role in adherence, and obtaining their willingness to actively participate in the treatment of their children is as important as it is for older patients who take full responsibility for administering their medications.

The present review identified multiple factors that should be considered for obtaining patient commitment to follow the intended treatment regimen. Other reviews ${ }^{7,11}$ have highlighted the complex interrelationship between factors that affect patient ability and motivation to adhere to treatment, and generally support the central position of patient willingness in our model. Our flow chart offers a reminder to physicians of the major factors involved in improving adherence. In particular, the chart highlights the importance of fostering a good patient-physician relationship, understanding patient beliefs, attitudes and perceptions of disease and treatment, and to provide the necessary education to improve patient understanding of their condition and the value of prescribed treatment. As well as prescribing safe and effective medications, obtaining the willingness of patients to participate in the management of their asthma is important for increasing adherence and improving the chance of successful therapy.

\section{Conclusion}

Patient adherence to asthma can be improved, and the likelihood of treatment success increased, by paying attention to the factors that influence patients' willingness to participate in their treatment. 
References

1. Schmier JK, Leidy NK. The complexity of treatment adherence in adults with asthma: challenge and opportunities. J Asthma 1998; 35: 455-72.

2. Royal Pharmaceutical Society of Great Britain. From compliance to concordance. Achieving shared goals in medicine taking. London: Royal Pharmaceutical Society of Great Britain, London, 1997.

3. Jones PW. Health status, quality of life and compliance. Eur Respir Rev 1998; 8: 243-6.

4. National Institutes for Health, National Heart, Lung and Blood Institute. Global Initiative for Asthma. Publication Number 96-3659B, 1998.

5. Bosley CM, Parry DT, Cochrane GM. Patient compliance with inhaled medication: Does combining B2-agonists with corticosteroids improve compliance? Eur Respir J 1994; 7: 504-9.

6. Braunstein GL, Trinquet G, Harper AE.

Compliance with nedocromil sodium and a nedocromil sodium/salbutamol combination. Eur Respir J 1996;

9: $893-8$.
7. van der Woude HJ, Aalbers R. Compliance with inhaled glucocorticoids and concomitant use of longacting ß2-agonists. Respir Med 2001; 95: 404-7.

8. Clark N, Jones P, Keller S, Vermeire P. Patient factors and compliance with asthma therapy. Respir Med 1999;93:856-62.

9. Vermeire PA, Rabe KF, Soriano JB, Maier WC. Asthma control and differences in management practices across seven European countries. Respir Med 2002;96:142-9.

10. Tettersell MJ. Asthma patients' knowledge in relation to compliance with drug therapy. J Adv Nurs 1993;18:103-13.

11. Clark NM, Gong M, Schork MA, Evans D et al. Impact of education for physicians on patient outcomes. Pediatrics 1998; 101: 831-6.

12. Chapman KR, Walker L, Cluley S, Fabbri L. Improving patient compliance with asthma therapy. Respir Med 2000;94:2-9. 